\title{
The processing and evaluation of news content on social media is influenced by peer-user commentary
}

\author{
Arnout B. Boot (iD ${ }^{1 凶}$, Katinka Dijkstra ${ }^{1}$ \& Rolf A. Zwaan ${ }^{1}$
}

Contemporary news often spreads via social media. This study investigated whether the processing and evaluation of online news content can be influenced by Likes and peer-user comments. An online experiment was designed, using a custom-built website that resembled Facebook, to explore how Likes, positive comments, negative comments, or a combination of positive and negative comments would affect the reader's processing of news content. The results showed that especially negative comments affected the readers' personal opinions about the news content, even in combination with other positive comments: They (1) induced more negative attitudes, (2) lowered intent to share it, (3) reduced agreement with conveyed ideas, (4) lowered perceived attitude of the general public, and (5) decreased the credibility of the content. Against expectations, the presence of Likes did not affect the readers, irrespective of the news content. An important consideration is that, while the negative comments were persuasive, they comprised subjective, emotive, and fallacious rhetoric. Finally, negativity bias, the perception of expert authority, and cognitive heuristics are discussed as potential explanations for the persuasive effect of negative comments.

\footnotetext{
${ }^{1}$ Erasmus University Rotterdam, Rotterdam, the Netherlands. ${ }_{\text {email: boot@essb.eur.nl }}$
} 


\section{Introduction}

he rise of social media has introduced virtually unlimited communication and unprecedented access to information. Information conveyed via social media is complemented with several social cues reflecting a variety of user interactions. Some examples are peer-user comments, Likes, favourites, recommendations, number of views, user-created content, user profiles, personal playlists, consumer reviews, and user ratings. Likes (i.e., favourites) and user comments are perhaps the most ubiquitous and universally implemented features across different social media platforms (e.g., Facebook, Twitter, Instagram). The current experimental study investigated how social media users are being affected by Likes and user comments when they process social media content. We used five distinct outcome variables: attitude, share intent, ideological congruence (i.e., level of agreement with the content), perceived public attitude, and credibility. These variables were partially adapted from previous studies (Appelman and Sundar, 2016; Winter et al., 2015; Xu, 2013).

Conformity behaviour in groups has been a key research topic in psychology. The early Asch paradigm studies have shown that individuals tend to change their judgements to conform to the majority group (Asch, 1951, 1955, 1956). In literature about opinion formation processes, conformity to public opinions has been referred to as the bandwagon effect (Nadeau et al., 1993). The bandwagon is a metaphor for a popular activity. This metaphor originates from nineteenth-century American parades, in which a wagon would carry a band playing music to attract followers (Schmitt-Beck, 2015). The bandwagon effect describes how individuals adapt their own opinions to conform to the public's opinions. This phenomenon has been referred to as a cognitive bias known as the bandwagon heuristic (Sundar, 2008), and has also been related to the consensus heuristic, the cognitive bias to perceive the majority's opinion as to the correct one (Mutz, 1998). On social media, Likes and user comments can be perceived as the metaphorical 'bandwagon', reflecting the popular attitudes and opinions among peer social media users. In turn, these social cues have been shown to persuade other users that observe these posts, an evident bandwagon effect. For example, Likes have previously been shown to increase the likeability of the content and the probability that the observer will also hit the Like button (Bak and Keßler, 2012; Sherman et al., 2016). The bandwagon effect predicts that social media users adapt their own beliefs, attitudes, and opinions to match that of their peers (Lowe-Calverley and Grieve, 2018; Kim, 2018; Sundar, 2008; Waddell, 2018a, 2018b). In the current study, we investigated whether Likes and comments can cause a bandwagon effect on the processing and evaluation of news content on a social networking site.

Likes. The Like button and similar social-media features (e.g., Favourite, Upvote, +1 ) have been conceptualized as paralinguistic digital affordances (PDAs); communicative cues within social media without a specific predefined meaning (Hayes et al., 2016). The Like button reflects a leading aspect of user engagement: a single-click social tool that, at the surface level, enables users to express enjoyment or share their interest in specific content. However, its affordance and meaning have been shown to extend its phatic design. For example, the Like button is also used for social support, to maintain social ties, and as a tool for impression management (Carr et al., 2016; Eranti and Lonkila, 2015; Hayes et al., 2016; Ozanne et al., 2017). Altogether, the Like button is a central feature of the social media environment. Likes are utilized and observed by billions of social media users, which emphasizes the importance of investigating its influence.

In contrast to previous studies, we compared the presence with the omission of Likes. Previous experimental studies have compared the effects of "low" and "high" numbers of Likes (e.g., Winter et al., 2015). However, the effects of inclusion and omission of Likes on user cognition have rarely been examined. In April of 2019, Instagram announced that the company was planning to hide the number of Likes from its users because it did not want Instagram "to feel like a competition" (Rodriguez, 2019). Facebook (owned by the same company) also started a trial of selectively omitting Likes to reduce the negative impact of social comparison ("Facebook to hide a number of likes", 2019). Considering these recent developments, we considered it especially relevant to investigate the cognitive implications of the omission compared to the presence of Likes. We hypothesized that Likes affect the observer's content processing and evaluation by creating a bandwagon effect. More specifically, we expected Likes to improve (a) attitude towards the content, (b) the likelihood that the reader will share or recommend the content, (c) agreement with ideas conveyed in the content, (d) the perceived public attitude, and (e) the credibility of the content (H1).

Peer-user comments. A second variable that we manipulated in our experiment was the presentation of user comments. Previous studies have shown that the presence of comments affects the perception and evaluation of online news articles. They can increase or decrease the acceptance and attitudes of the reader (Kim, 2018; Waddell, 2018a, 2018b; Winter et al., 2015). As with Likes, the psychological mechanism for the effect of comments has been framed in terms of the bandwagon heuristic (Sundar, 2008; Waddell, 2018b). Online news readers use peer comments as cues that are representative of the general public's opinion, which influences their own attitude towards the content (Kim, 2018). Furthermore, comments can lower the perceived credibility when they are used as a quote or a source of evidence within a news article (Waddell, 2018a). However, if comment sections include uncivil language, they can increase the perceived credibility of the main article due to a contrast effect (Thorson, Vraga, and Ekdale, 2010).

Peer comments provide additional information to the reader, which can consist of objective or subjective content. An objective comment is factual, based on evidence and logic, or verifiably true or false. An example

"Greta Thunberg is a young environmental activist because, as a teenager, she has approached and challenged multiple world leaders to mitigate climate change". (Example 1)

A subjective comment, on the other hand, is based on opinions or personal anecdotes, which cannot be independently verified or refuted. Hence, subjective comments are not necessarily grounded in reality. An example:

"Greta Thunberg is a hero. She's brave enough to do what many people are thinking but are too afraid to act on. I hope more world leaders take her message seriously." (Example 2)

Hinnant et al. (2016) investigated the effects of anecdotal versus scientific evidence in comments on an article about climate change. As might be expected, comments that invoked scientific evidence had a greater influence on the readers' attitude than comments that invoked anecdotal evidence. However, for conservative readers with low need for cognition (a construct that represents the joy of engaging in one's own thought processes), even anecdotal rhetoric was found to influence the perceived credibility and disrupt the intended message of the story (Cacioppo and Petty, 1982). Further examination of this susceptibility to subjective comments is crucial to understanding 
online behaviour and cognition in an era of mounting 'fake news', misinformation, and disinformation, where anyone can join online debates or add commentary to websites' comment sections (Lazer et al., 2018). Therefore, in addition to Likes, we investigated the effect of different comment types on the observer.

In this study, participants encountered either positive, negative, no comments or a combination of positive and negative comments. These comments comprised subjective statements (as shown in Example 2), emotive language and/or fallacious rhetoric. We hypothesized that the reader's content processing and evaluation would be affected by the presence of these comment sections. Based on a bandwagon effect, we expected positive comments would improve whereas negative comments would impair (a) attitude towards the content, (b) the likelihood the reader will share or recommend the content, (c) agreement with ideas conveyed in the content, (d) the perceived public attitude, and (e) the credibility of the content $(\mathrm{H} 2)$.

Similar to the current study, Winter and colleagues (2015) investigated the effects of peer comments and Likes on Facebook news channels and the related psychological mechanisms of information processing. In their online experiment, participants saw a summary of an online news story on a Facebook page including comments and Likes. The type of comments and the number of Likes were manipulated in a $5 \times 2$ between-subjects design. The comment types were either positive or negative and either subjective or argumentative (i.e., objectively and independently verifiable or refutable arguments), which rendered four different comment conditions including the fifth condition without comments. The number of Likes was either "low" (around 40) or "high" (around 500). Subsequently, participants read the original article after which they answered questions about their attitude toward the topic, perceived public opinion, evaluation of the article (i.e., writing style, usefulness, and likeability), and credibility of the text.

Against the authors' expectation of bandwagon heuristics, no conformity effects of Likes were found. In other words, the number of Likes did not influence the readers' evaluation of the news article. Negative comments were found to be more persuasive than Likes and positive comments. The authors explained this based on a ceiling effect; readers had a high level of agreement with the ideas conveyed in the article. Therefore, information of negative valence aroused more attention (Winter et al., 2015). Thus, positive comments as well as Likes (i.e., a positive expression) did not show further strengthening effects. The authors suggested that this interpretation could be tested with a variation of the slant of the article (i.e., ideas conveyed in the article), without making further suggestions for the kind of content. In their experiment, the article that was presented to the readers described arguments from a professor in economics against the prohibition of marijuana, proposing that legalization of the drug would lead to more control. The slant of the content was ideologically progressive and liberal. Therefore, the content may have caused high levels of agreement in participants, comprising mainly European academic students.

Hypothetically, if a high agreement with the content decreases the influence of Likes and positive comments due to a positive ceiling effect, then initial disagreement with the content could potentially prevent a positive ceiling effect and increase the influence of Likes and positive comments. Therefore, we expected that Likes and positive comments would have a greater influence on the reader's attitude towards social-media content if the reader has a negative predisposition as compared to a positive predisposition towards the content (H3).

The relatively small effects of positive comments and likes as compared to negative comments in the study of Winter and colleagues could also be ascribed to a negativity bias. Previous studies have shown the prevalence of negativity bias; generally, negative information induces stronger psychological effects than neutral and positive information (Baumeister et al., 2001; Ito et al., 1998; Norris, 2021; Rozin and Royzman, 2001). Therefore, we expected that negative comments in the current experiment should have a greater influence on the reader than positive and neutral comments. Furthermore, we expected that this negativity bias would be persistent across different ideological dispositions towards the article. More specifically, negative comments should have a greater influence on the reader's attitude towards socialmedia content than positive comments, irrespective of the reader's personal disposition towards the content (H4).

Another explanation for the lack of strengthening effects of Likes in the study by Winter and colleagues might be related to the experiment's ecological validity. Participants viewed a static and non-interactive screenshot of a Facebook page. Arguably, the social cues on this static image were less meaningful to the user than social cues in an interactive social-media interface. In addition, a static image of a Facebook page may induce unnatural behaviour. For instance, participants are more likely to secondguess the experimental manipulation and the experimenter's hypothesis. To ensure the ecological validity of the current experiment, participants performed the task using an ostensibly real and interactive social-media interface.

Content types. In the current study, we compared the effects of Likes and comments between different content types. Specifically, the article contents would engender a variety of attitudes in the readers: either agreeing, disagreeing or neutral dispositions. Considering the cohort and background of the subject pool for the current study (psychology students at Erasmus University Rotterdam) it was assumed that they tend to have more liberal than conservative ideologies (Inbar and Lammers, 2012; Pohl et al., 2021). This assumption has led to the selection of three news reports, each assumed to induce a certain attitude in the participant:

(1) Ideologically congruent: A news article about a meeting between climate activist Greta Thunberg and Canadian Prime Minister Justin Trudeau. We assumed that most participants would agree with Greta Thunberg's climate activism. Therefore, participants would predominantly have a positive predisposition towards the content of this article.

(2) Ideologically incongruent: A news article about a study reporting a relationship between violent video games and aggressive behaviour. Considering the cohort and age of the participants, they were assumed to have more progressive ideas and to be likely to have a personal affinity for video games (e.g., $92 \%$ of people between the ages 16 and 24 years play video games in the UK; see "Gaming penetration in the United Kingdom (UK) from 2013 to 2021, by age group and gender", 2021). Thus, participants were assumed to have a more negative predisposition towards the content of this article.

(3) Ideologically neutral: A news article about an upcoming tropical storm near the coast of Ireland. This article described a mere weather phenomenon, which did not convey an explicit ideology.

Comparing the effect of Likes and peer comments between different types of content would yield comprehensive findings. Specifically, it would show how Likes and peer comments affect information processing under different reader dispositions. Accordingly, we hypothesized that if the article content is ideologically neutral, then the reader would neither agree nor disagree with the content. Therefore, likes and comments should 


\section{Like 576 people like this}

\section{Comments}

\section{John_walker}

Greta Thurnberg is a hero. She's brave enough to do what many people are thinking but are too afraid to act on. I hope more world leaders take her message seriously.

\section{SarahLeandra09}

She's nothing special. What did she do? Skip school to protest? She doesn't have radical ideas.

\section{HaroldMinster4u}

I really admire Greta's actions, it's amazing what she has accomplished already. More officials will endorse her ideas if we spread the message.

d) 14

\section{JessEwan85}

Just another flavor of the month for your morning news. I don't blame her, she's just a puppet in all this.

\section{View more comments...}

Add a comment...

Submit

Fig. 1 Example of the mixed comment section and Likes manipulations. This comments section manipulation was presented to participants in group 5 (see Table 1). These comments and Likes (both under the article and under the comments) were presented below a news article about Greta Thunberg. The comments only comprised subjective rhetoric.

have a greater influence (i.e., bandwagon effect) on the reader's attitude than if the article content conveys an ideology. Thus, Likes and comments should have a greater influence on the reader's attitude towards ideologically neutral content as compared to content with an ideological slant (H5).

The experiment. A custom website, using a similar interface as Facebook, was designed for this study. This site contained news articles and complementary social cues. A copy of the original website can be viewed online (https://online-task-anonymousexample.netlify.com/). We highlight five key features of the current design: (1) The experiment compared the presence of Likes with the omission of Likes, investigating the effect of the contemporary trend of omitting Likes in social media platforms. (2) The Likes were added to the original article, but they were also added to the comments, as often seen in social media formats. (3) The comments only comprised subjective rhetoric (see Example 2 and Fig. 1), no objective comments such as Example 1 were presented. (4) To investigate the effect of the overall valence or sentiment of the comments, four conditions were compared: positive comments, negative comments, positive and negative (mixed) comments, and no comments (control). (5) To examine the interaction between comments and the reader's ideological disposition, three different news articles were used: an ideologically congruent article, an ideologically incongruent article, and an ideologically neutral article.
The current experiment resembles the research design by Winter et al. (2015), however, there are some key methodological differences and extensions. Firstly, we used an interactive htmlbased website that participants could browse on their personal computer, simulating a more personal online experience. The website contained more realistic interactive elements such as selectable texts, navigation bars, buttons, and cover image slideshows. Secondly, multiple news contents were presented as opposed to one news article, which was assumed to create a variety of predispositions within the readers, that in turn, could interact differently with the complementary Likes and comments. Furthermore, the comment manipulation in the current experiment also included an additional condition that was not included by Winter et al., a mixed comments condition including both positive and negative comments. The Likes manipulation was different in two aspects: (1) comparing the presence of Likes with the omission of Likes rather than comparing a high and a low number of Likes, and (2) the Likes were presented under the main news article as well as under each individual comment. Finally, in addition to the outcome measures ideological congruence, perceived public attitude, and credibility, which are comparable to the measures used by Winter et al., we also added share intent and general attitude.

Outcome variables. After reading each article, participants rated a set of statements reflecting five different categories: attitude, 
share intent, ideological congruence, perceived public attitude, and credibility (see Table 2 for an overview of the items). These variables were partially adapted from previous studies (Appelman and Sundar, 2016; Winter et al., 2015; Xu, 2013): (a) attitude-the reader's personal disposition towards the content, (b) share intent -the reader's intension to share or recommend the content, (c) ideological congruence- the extent to which the reader agrees with content-conveyed ideas, (d) perceived public attitude-the reader's perception of the general public's attitude towards the content, and (e) credibility - the reader's perception of the credibility of the content. In this study, these categories were considered to adequately reflect content processing and evaluation.

\section{Methods}

Preregistration. This study was preregistered on Open Science Framework. We preregistered our hypotheses, study design, sample size, analysis type, exclusion criteria, and statistical inference criteria ${ }^{1}$.

Participants. This study was approved by the Ethics Review Committee DPECS at Erasmus University Rotterdam (application reference 19-043). All participants provided their informed consent before participating in this study. A total of 560 students, first and second-year bachelor of Psychology students from Erasmus University Rotterdam, participated in the experiment. Participants were excluded from the analysis if their time spent on the reading task was shorter than two minutes, which ensured all participants in the analysis carefully read the article. Moreover, participants were excluded if they did not complete the questionnaire. In addition, data from four participants were removed because they used a mobile operating system to perform the task. The final sample size comprised 412 participants (330 females, Mean $_{\text {age }}=20.52, \mathrm{SD}=2.85$ ). Demographic details of the sample can be found in Supplementary Table S1.

Design and materials. The experiment was an online task that participants could perform on their own computers. We designed and built a website that was ostensibly similar to the graphical user interface of Facebook and other social networking sites (for an interactive example see the link in Table 1). Three news articles were presented on this website: an article about climate activist Greta Thunberg, an article about a tropical storm near the coast of Ireland, and an article about a study that found a relationship between violent video games and behaviour. The latter article was slightly altered so that it more strongly conveyed the idea that this relation was true, leaving out subtle nuances that were in the original article. The three news articles were adapted from the English newspaper the Guardian and showed consistent writing style and structure. Each article reflected a different content type (within-subject variable): ideologically congruent,

\begin{tabular}{|c|c|c|}
\hline $\begin{array}{l}\text { Between-subject } \\
\text { conditions }\end{array}$ & Likes presented & Likes omitted \\
\hline Positive comments & Group $1(\mathrm{dprz})^{\mathrm{a}}$ & Group 2 (wgdy) \\
\hline Negative comments & Group 3 (fspc) & Group 4 (pdrt) \\
\hline $\begin{array}{l}\text { Mixed (positive and } \\
\text { negative comments) }\end{array}$ & Group 5 (rdts) & Group 6 (wphj) \\
\hline No comments & Group 7 (ypdt) & Group 8 (cnfp) \\
\hline
\end{tabular}

Note: This is an overview of the eight between-subject conditions.

aEach condition can be viewed using the unique code on the example website https://onlinetask-anonymous-example.netlify.com/ or they can be viewed as non-interactive examples in Supplementary Materials. Each participant browsed three different news articles. neutral, and incongruent. The articles were complemented with two types of social cues: Likes and user comments, which were manipulated to one of eight between-subject conditions (see Table 1). The mixed comments condition, presented in Fig. 1, comprised a combination of two positive and two negative comments, which were identical to half of the comments in the positive and negative comments conditions. An interactive example of the experiment, containing all Web elements, can be viewed at https://online-task-anonymous-example.netlify.com/. Each experimental condition can also be viewed in Supplementary Materials (non-interactive pdf format). The Likes were presented both below the news article and under each comment as shown in Fig. 1.

Procedure. Participants were randomly assigned to one of eight conditions and were instructed to use a computer and minimize potential distractions and disturbances. They received a link to the experiment and a complimentary code to start the task. To start the task, participants provided their informed consent and entered a specific four-letter code. After an introductory page, participants read three news articles, each followed by an embedded Qualtrics software survey. The order of within-subject conditions (i.e., article-content type) was based on block randomization, beginning with either congruent or incongruent content types, followed by the neutral content type, and ending with either the congruent or incongruent content type. Subsequently, the participants filled out a questionnaire regarding personal social-media usage and demographic traits. Finally, a debriefing page was used to inform participants about the diminished veracity of the altered article about violent video games and aggression.

Measures. Five outcome variables, reflecting content processing and evaluation, were assessed using seven-point scale ratings, see Table 2 for an overview of the items.

Analysis. We used a sequential-analyses design (Lakens, 2014). This means we performed an interim analysis using predetermined sample size and decided to end data collection based on the predetermined inference criteria (see preregistration on OSF at https://osf.io/d37f5). We corrected for alpha inflation using the O'Brien-Fleming procedure, alpha $=0.011$. Mixed factorial ANOVAs $(2 \times 4 \times 3)$ were performed for each outcome variable, testing main effects and interaction effects of Likes (between-subjects; 2 levels; omitted vs. presented), comments (between-subjects; 4 levels; positive, negative, mixed, and no comments), and article-content type (within-subjects; 3 levels; congruent, incongruent, and neutral). Mauchly's test was used to determine whether the assumption of sphericity was violated. Greenhouse-Geisser estimates of sphericity were used to correct the degrees of freedom. Article-content type was a within-subjects variable that violated the sphericity assumption across all five outcome variables (attitude, $W=0.977, p=0.009, \varepsilon=0.98$; share intent, $W=0.977, p=0.008, \varepsilon=0.98$; ideological congruence, $W=0.935, \quad p<0.001, \quad \varepsilon=0.94 ;$ perceived public attitude, $W=0.983, p<0.001, \varepsilon=0.98$; credibility, $W=0.896 p<0.001$, $\varepsilon=0.91)$. Bonferroni corrections were used to adjust $p$-values for multiple comparisons.

\section{Results}

We report five mixed factorial ANOVAs post-hoc analyses for each of the outcome variables attitude, share intent, ideological congruence, perceived public attitude, and credibility. 
Table 2 Outcome variables with internal consistencies, descriptions, and items.

\begin{tabular}{|c|c|c|}
\hline $\begin{array}{l}\text { Variable; } \\
\text { Internal consistency }\end{array}$ & Description & Items (i.e., statements) \\
\hline $\begin{array}{l}\text { Personal attitude; } \\
\text { Cronbach's alpha }=0.81\end{array}$ & Disposition of the reader towards the article & $\begin{array}{l}\text { "I feel positive about the article" } \\
\text { "I consider this news to be valuable" } \\
\text { "I like this article" }\end{array}$ \\
\hline $\begin{array}{l}\text { Share intent } \\
\text { Cronbach's alpha }=0.86\end{array}$ & Expected behavioural/social response & $\begin{array}{l}\text { "I would recommend this article to my friends" } \\
\text { "I would hit the Like button" } \\
\text { "I would share this article with my friends" }\end{array}$ \\
\hline $\begin{array}{l}\text { Ideological congruence } \\
\text { (ideologically congruent } \\
\text { article); Cronbach's } \\
\text { alpha }=0.77^{\star}\end{array}$ & $\begin{array}{l}\text { The extent to which the reader agrees with } \\
\text { ideas conveyed in the article about climate } \\
\text { activist Greta Thunberg }\end{array}$ & $\begin{array}{l}\text { "Greta Thunberg is doing great work as an environmental activist" } \\
\text { "Greta Thunberg's meeting with Canadian prime minister, Justin Trudeau } \\
\text { was a success" } \\
\text { "Justin Trudeau will act on climate and support Great Thunberg's cause' }\end{array}$ \\
\hline $\begin{array}{l}\text { Ideological congruence } \\
\text { (ideologically neutral article); } \\
\text { Cronbach's alpha }=0.77^{\star}\end{array}$ & $\begin{array}{l}\text { The extent to which the reader agrees with } \\
\text { ideas conveyed in the article about a } \\
\text { tropical storm }\end{array}$ & $\begin{array}{l}\text { "Hurricane Lorenzo is dangerous" } \\
\text { "People should prepare for Hurricane Lorenzo" } \\
\text { "People should be concerned about the potential impact of Hurricane } \\
\text { Lorenzo" }\end{array}$ \\
\hline $\begin{array}{l}\text { Ideological congruence } \\
\text { (ideologically incongruent); } \\
\text { Cronbach's alpha }=0.77^{\star}\end{array}$ & $\begin{array}{l}\text { The extent to which the reader agrees with } \\
\text { ideas conveyed in the article about violent } \\
\text { videogames and aggression }\end{array}$ & $\begin{array}{l}\text { "Violent video games induce aggressive behaviour" } \\
\text { "Kids who play violent video games think violence is acceptable in real } \\
\text { life" } \\
\text { "Parents should limit their kids' exposure to violent video games" }\end{array}$ \\
\hline $\begin{array}{l}\text { Perceived public attitude } \\
\text { (i.e., bandwagon perception); } \\
\text { Cronbach's alpha }=0.85\end{array}$ & $\begin{array}{l}\text { The reader's perception of the general } \\
\text { public's opinion about the article }\end{array}$ & $\begin{array}{l}\text { "The general public feels positive about this article" } \\
\text { "The general public considers this news to be valuable" } \\
\text { "The general public likes this article" }\end{array}$ \\
\hline $\begin{array}{l}\text { Credibility; Cronbach's } \\
\text { alpha }=0.81\end{array}$ & $\begin{array}{l}\text { The readers perception of the credibility of } \\
\text { the article's content }\end{array}$ & $\begin{array}{l}\text { "How well do the following words describe the article's content?"- } \\
\text { "Accurate" - "Authentic" - "Believable" (Three separate items) }\end{array}$ \\
\hline
\end{tabular}

Note: Seven-point-scale ratings were used for the items. Each of the five categories was measured separately for each article; the items were identical across articles, except for the category ideological congruence.

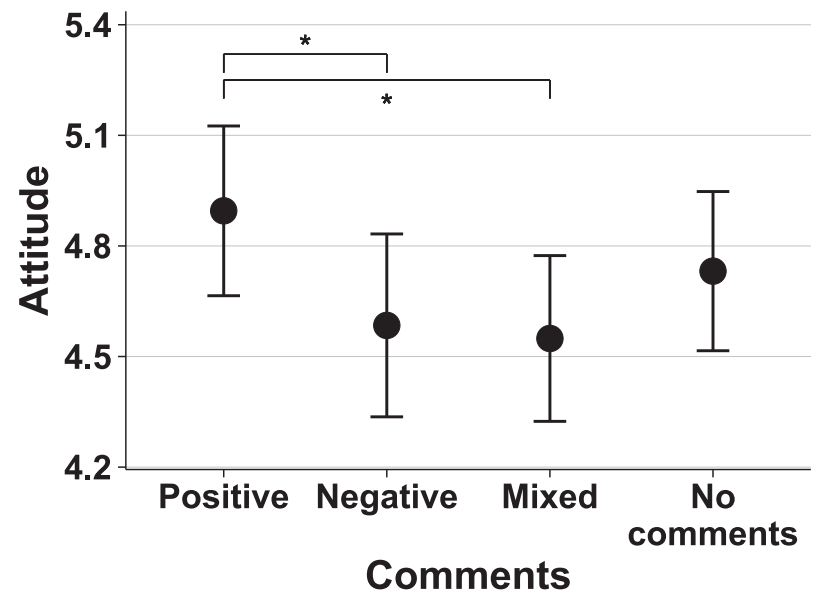

Fig. 2 Main effect of comment sentiment on attitude. Participants viewed news content on a manipulated but ostensibly real social networking site. We manipulated the sentiment of user comments to be either positive, negative, both (i.e., 'mixed'), or no comments. Error bars represent $95 \%$ Cls, significant differences $(p<0.011)$ are indicated with asterisks.

Attitude. We found a significant main effect of comment sentiment on attitude, $F(3,404)=4.19, p=0.006$, generalized $\eta^{2}=0.013$. Pairwise comparisons revealed a significant difference between positive and mixed comments, $p=0.002$, and a significant difference between positive and negative comments, $p=0.008$. Figure 2 shows that negative and mixed comment sentiments (i.e., negative and positive comments) induced more negative attitudes towards the article content as compared to positive comments. Moreover, we found an interaction effect (although above the alpha level of 0.011 ) between article-content type and comments, $F(6.14,826.70)=2.39, p=0.028$, generalized $\eta^{2}=0.010$. This interaction effect can be seen in Fig. 3: without comments, participants were relatively more positive about the

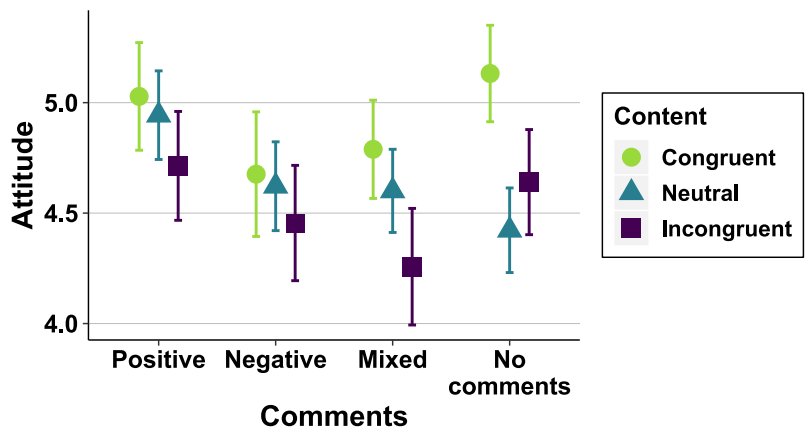

Fig. 3 Interaction effect of comment sentiment and article-content type on attitude. Participants viewed three news articles on a manipulated but ostensibly real social networking site. The ideas conveyed by the article contents were assumed to be congruent, neutral, and incongruent with the participants' ideological dispositions. Error bars represent 95\% Cls.

congruent article and relatively more negative about the neutral article. Finally, we found a significant main effect of articlecontent type on attitude, $F(2.05,826.70)=13.21$, $p<0.001$, generalized $\eta^{2}=0.018$ (see Supplementary Fig. S1), but there was no effect of the Likes condition on attitude, $F(1,404)=0.83$, $p=0.362$.

Share intent. There was a significant main effect of comments on share intent, $F(3,404)=4.52, p=0.006$, generalized $\eta^{2}=0.004$. Pairwise comparisons revealed a significant difference between positive and mixed comments, $p=0.002$, and a significant difference between positive and negative comments, $p=0.003$. However, the difference between no comments and mixed was nonsignificant, $p=0.013$ (pre-determined alpha level was 0.011 ). Similarly, no comments and negative comments were not significantly different either, $p=0.019$. Figure 4 shows that readers were considerably less likely to share the article if it was 


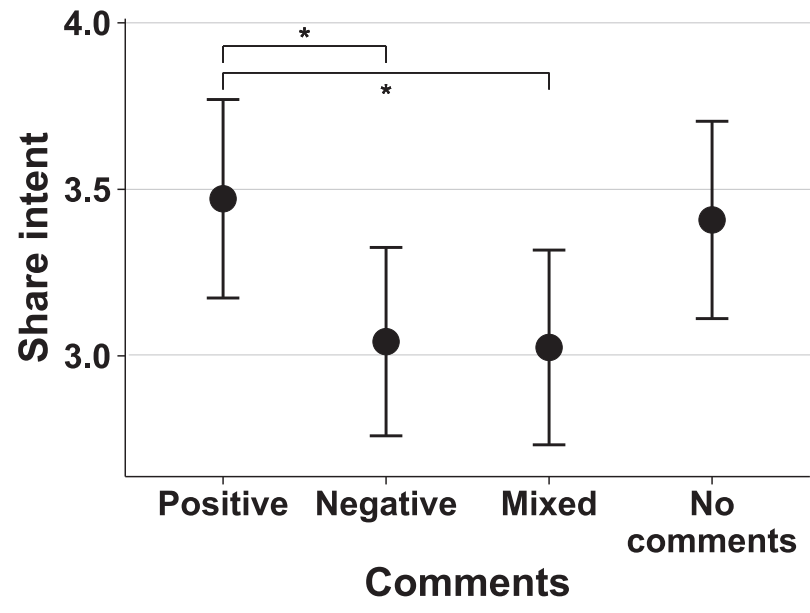

Fig. 4 Main effect of comment sentiment on share intent. Error bars represent $95 \% \mathrm{Cls}$, significant differences $(p<0.011)$ are indicated with asterisks.

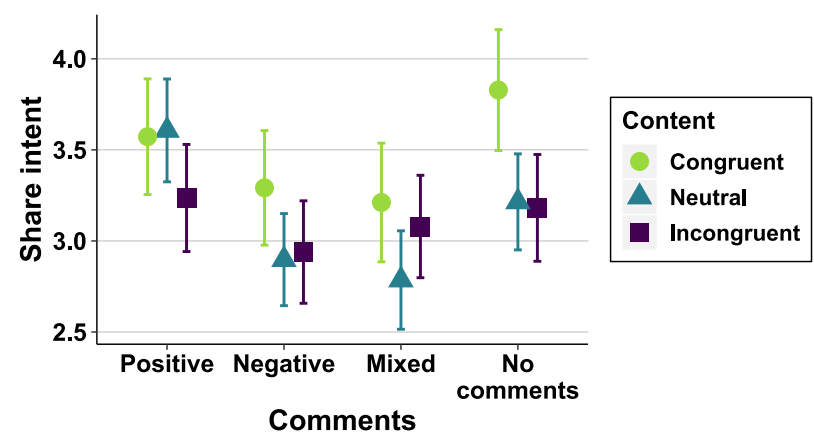

Fig. 5 Interaction effect of comment sentiment and article-content type on share intent. Error bars represent $95 \%$ Cls.

complemented with negative or mixed comments as compared to positive comments. Furthermore, we found an interaction effect (above the alpha level of 0.011) between article-content type and comments, $\quad F(6.14, \quad 826.91)=2.16, \quad p=0.045, \quad$ generalized $\eta^{2}=0.007$. Figure 5 shows that the overall presence of comments decreased the willingness to share the congruent article content compared to no comments. Finally, we found a significant main effect of article-content type on share intent, $F(2.05$, $826.91)=11.60, p<0.001$, generalized $\eta^{2}=0.013$ (see Supplementary Fig. S1), but there was no effect of the Likes condition on share intent, $F(1,404)=0.09, p=0.761$.

Ideological congruence. There was a significant main effect of comments on ideological congruence, $F(3,404)=12.15$, $p<0.001$, generalized $\eta^{2}=0.039$. Pairwise comparisons showed significant differences between all comment types, $p<0.001$, except for positive and no comments, and negative and mixed comments. In Fig. 6 can be seen that for both negative and mixed comments the readers showed less agreement with ideas conveyed in the article as compared to positive comments or no comments. Moreover, we found a significant main effect of article-content type on ideological congruence, $F(2.13$, 860.51 ) $=103.07, p<0.001$, generalized $\eta^{2}=0.123$ (see Supplementary Fig. S1). However, we did not find a significant interaction effect between article-content type and comments, $F(6.39$, $860.51)=1.89, p=0.079$, and there was no significant main effect of Likes on ideological congruence, $F(1,404)<0.01, p=0.995$.

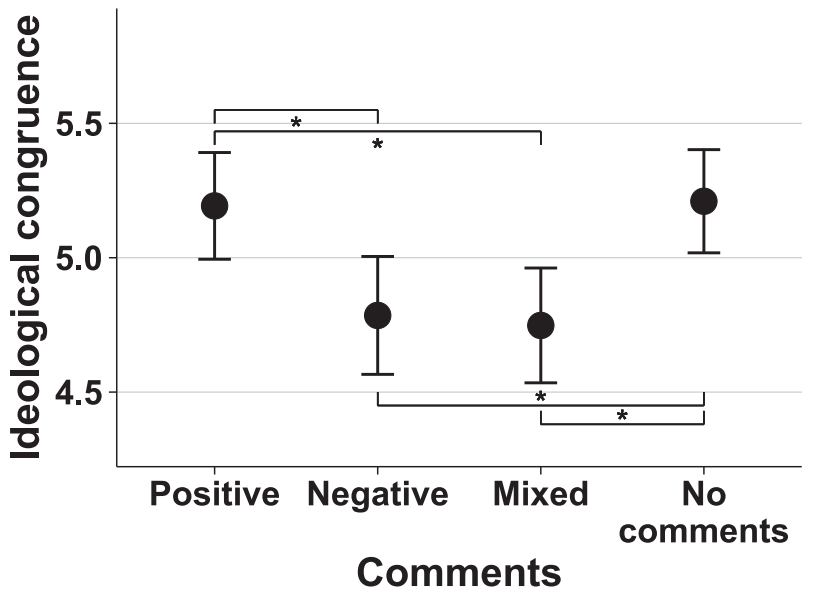

Fig. 6 Main effect of comment sentiment on ideological congruence. Error bars represent $95 \% \mathrm{Cls}$, significant differences $(p<0.011)$ are indicated with asterisks.

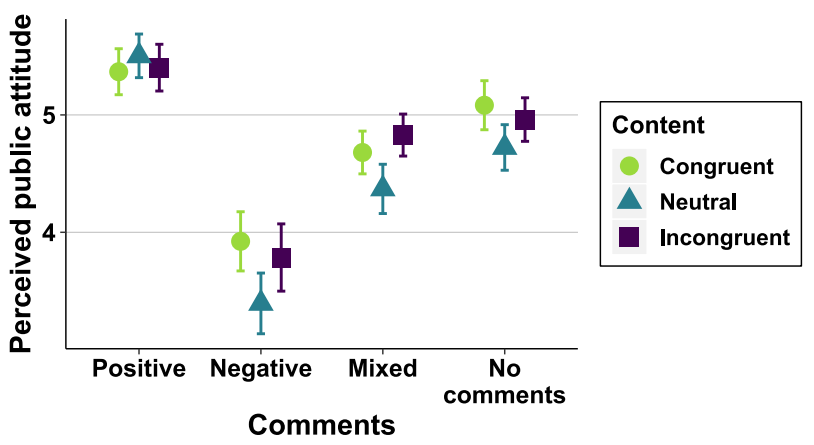

Fig. 7 Interaction effect of comment sentiment and article-content type on perceived public attitude. Error bars represent $95 \% \mathrm{Cls}$.

Perceived public attitude. We found a significant main effect of comments on perceived public attitude, $F(3,404)=87.22$, $p<0.001$, generalized $\eta^{2}=0.248$, and a significant interaction effect between article-content type and comments, $F(6.10$, $821.93)=3.07, p=0.006$. Figure 7 shows that comment sentiment had a greater effect when complementing the neutral article-content. Furthermore, pairwise comparisons of the comment conditions revealed significant differences between all comment types, $p \leq 0.007$. Figure 7 shows that the perceived public attitude was highly affected by comment sentiment. Positive comments induced the most positive perceived public attitude and negative comments induced the most negative perceived public attitude. Finally, we found a significant main effect of article-content type on the perceived public attitude, $F(2.03$, 821.93) $=10.01, p<0.001$, generalized $\eta^{2}=0.012$, but there was no effect of the Likes condition, $F(1,404)=0.21, p=0.644$.

Credibility. We found a significant main effect of comments on credibility, $F(3,404)=8.68, p<0.001$, generalized $\eta^{2}=0.030$. Pairwise comparisons of the comments showed significant differences between all comment types, $p \leq 0.001$, except for positive and no comments, and negative and mixed comments. Figure 8 illustrates how the credibility of the article was affected by the presence of a comment section: both negative and mixed comments decreased the credibility as compared to positive comments or no comments. Moreover, we found a significant main effect of article-content type on the credibility, $F(2.21,891.72)=77.97$, $p<0.001$, generalized $\eta^{2}=0.092$ (see Supplementary Fig. S1). 


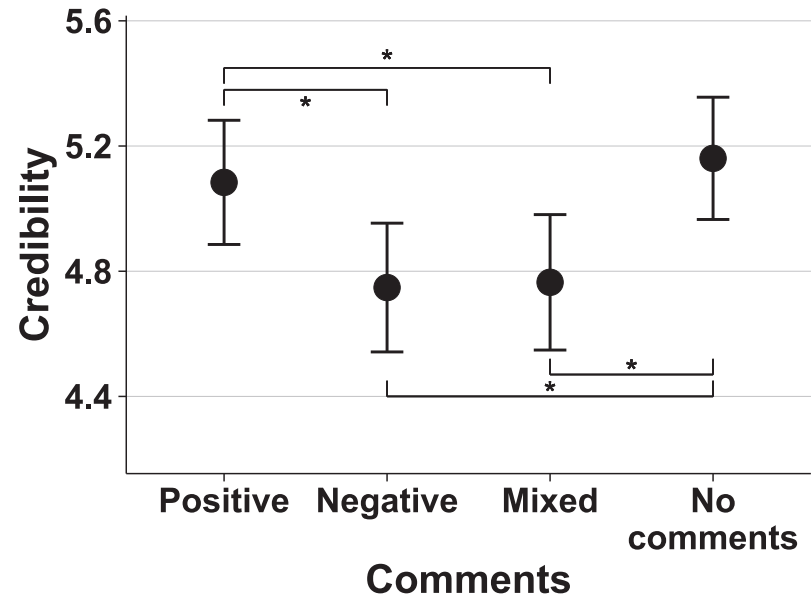

Fig. 8 Main effect of comment sentiment on credibility. Error bars represent $95 \% \mathrm{Cls}$, significant differences $(p<0.011)$ are indicated with asterisks.

However, we did not find a significant interaction effect between article-content type and comments, $F(6.62,891.72)=1.55$, $p=0.159$, and there was no significant main effect of Likes, $F(1,404)=1.41, p=0.236$.

\section{Discussion}

We investigated how Likes and user comments influenced the readers' processing and evaluation of news articles on social media. The comment sections, manipulated in our online experiment, affected the readers' content processing and evaluation. Particularly, the presence of negative comments (1) negatively affected the readers' attitude towards the news article, (2) reduced the likelihood the reader would share or recommend it, (3) reduced agreement with ideas conveyed in the article, (4) engendered the perceived public attitude more negatively, and (5) even reduced the credibility of the article. Importantly, participants were not instructed to read or utilize the comment sections to form their opinions. Nevertheless, their opinions were, in fact, influenced by comments comprising subjective, emotive, and even fallacious rhetoric.

In the current study, the presence of Likes had no effect on the readers' content evaluation. Thus, we found no evidence to support our first hypothesis (H1). This means we did not observe a bandwagon effect of the Likes. However, in line with our second hypothesis ( $\mathrm{H} 2)$, we found that negative comments had a negative effect on the readers' content evaluation. Analyses of the main effects of comment sentiment showed positive comments did not affect the reader as much as negative comments did (see Fig. 2). However, analyses of interaction effects between article-content type and comment sentiment revealed positive comments had different effects based on the predisposition towards the article (see Figs. 3 and 5). Particularly, the group that was presented with positive comments below the ideologically congruent article (i.e., news article about Greta Thunberg) showed relatively more negative attitudes and less intention to share the content compared to the group that was not presented with comments. However, when the ideologically incongruent article (i.e., news article about the relation between videogames and aggression) was presented with positive comments the effects were much smaller. Hence, our third hypothesis (H3), that a negative predisposition would increase the effect of positive comments compared to a positive predisposition, was not directly supported. That said, we did find a weak interaction between article content type and comment sentiment. That is positive comments induced larger positive effects in combination with the ideologically neutral article (i.e., news article about a tropical storm). This suggests that if the content does not convey a specific ideology, readers tend to rely more on the positive sentiment of the comments to form their opinions. Thus, peer comments can induce a bandwagon effect in readers who lack a strong predisposition. In that case, readers employ a consensus heuristic and use the majority opinion as a proxy for their own opinion (Mutz,1998; SchmittBeck, 2015).

The results show a pattern of negativity bias (Baumeister et al., 2001; Ito et al., 1998; Norris, 2021; Rozin and Royzman, 2001); even when readers were exposed to both positive and negative comments, they were more influenced by the negative comments, showing similar results as readers who were only exposed to negative comments. Therefore, we can conclude negative comments have a greater effect on the readers' attitude as compared to positive comments (H4). An important consideration is that the sample consisted of young adults; this age group has been shown to be more likely to have a negativity bias. Older adults, on the other hand, are likely to have a positivity bias (Carstensen and DeLiema, 2018). Future research could investigate whether older adults are less influenced by negative comments when they process social media content and whether they are more influenced by positive comments.

The mixed comments condition results provide unique implications; readers were exposed to two positive and two negative comments (i.e., two comments from the positive comments condition and two comments from the negative comments condition). The mixed comment section was qualitatively distinct from the purely negative and purely positive comment sections because it provided more contrasting peer opinions. This distinction explains the differentiation in the perceived public attitude, which was intermediate compared to the other conditions (see Fig. 7). However, the other outcome variables reflecting personal opinions (i.e., attitude, share intent, agreement with ideas, and credibility) were negatively biased, resembling the negative comments condition (see Figs. 2, 4, 6, and 8). This finding provides a unique dissociation of potential cognitive processes underlying the influence of comments. That is, the bandwagon heuristic is not an appropriate underlying cognitive process because there was no straightforward majority opinion in the mixed comments condition. A negativity bias, on the other hand, is a more appropriate cognitive mechanism for the finding that readers' personal opinions were relatively negative, even when half of the comments were positive. The results suggest that the evaluation and influence of the comments in the mixed condition were similar to how those same comments were evaluated in the other conditions; positive comments had little influence whereas negative comments greatly influenced the readers.

Based on our findings, we can conclude that especially comments with a negative sentiment can influence how readers evaluate the alluded content. A negativity bias explains how negative comments either aroused more attention and/or how negative comments received more conscious processing than positive comments (Baumeister et al., 2001). However, a negativity bias offers an evolutionary-based explanation for this finding. In theory, a negativity bias would increase a species' chance for survival, as a bias towards negative stimuli would improve the species' ability to avoid harmful situations (Norris, 2021). In addition to the evolutionary-based negativity bias, we present a more proximal explanation as well. Specifically, negative sentiment may be more interesting to the reader. Perhaps, encountering a criticism invokes a different, more contrasting, perspective on the content as compared to a mere endorsement. 
A criticism counters a previous idea, so it may appear as more novel and distinct (e.g., novel popout effect see Johnston et al., 1990), and thus, is perhaps more likely to be influential than a positive comment.

An unexpected finding was that the negative comments did not have a larger effect on the readers' attitude in combination with the neutral article as compared to the incongruent and congruent articles (H5). Interestingly, the presence of comments appeared to decrease the range of attitudes for the different articles and even decreased the readers' willingness to share or recommend the ideologically congruent article. This may be rooted in the possibility that, when readers are willing to share the original news content, they are more reluctant to share it if they associate the content with the subjective comment section.

Participants were asked to estimate the general public's attitude towards the articles. Not surprisingly, the comment sentiments were congruent with the perceived public attitudes, because the comment sections could be utilized as cues representing the general public's opinions. An important finding here is that participants were, in fact, aware of the positive comments in the mixed condition, showing more positive perceived public attitudes as compared to the negative sentiment condition (see Fig. 7). It is remarkable that the negativity bias was evident in reports of personal attitude but not in reports of the perceived public attitude. This difference suggests that while readers are more influenced by negative comments than positive comments, this is likely not due to an attentional bias towards negative cues. Otherwise, the perceived public attitude would also have shown a negativity bias. Instead, the negativity bias was probably caused by a distinction in the way negative and positive comments are processed.

The ostensible significance of a peer comment, and therefore its influence, may be based on the way the reader perceives this peer. For example, critical peer commenters may be perceived as more knowledgeable, more experienced, or more rational as compared to commenters who merely endorse the content. Therefore, criticisms or negative comments may be more persuasive than positive comments. The notion that readers attribute characteristics to the creators of the comments remains speculative. However, there is evidence that messages with negative sentiment are more convincing than those with a positive sentiment; Habernal and Gurevych (2016) created and tested a computational deep learning method to predict the convincingness of arguments on the Web. They found that in some cases arguments with a strong negative sentiment were more convincing than other arguments. Another study, performed by Kluck et al. (2019), showed that negative comments that expressed concerns about the veracity of a news story, diminished the perceived credibility of this news story. A negative comment is likely to convey criticism. Criticism might induce the perception of either intellectual or experience-based authority. In turn, the reader might engage in the heuristic cognition known as authority bias (Milgram, 1963), attributing greater accuracy or value to this comment than more positive comments. The notion that negative comments are more convincing or persuasive due to perceived authority by the reader should be investigated in future research.

We did not find evidence to support our first hypothesis, the presence of Likes did not affect the readers' content processing and evaluation. A possible explanation is that Likes are not as meaningful to an observer as they would be to the sender or the recipient. Furthermore, Likes could become more meaningful to the reader when they reflect user interactions within a familiar group such as friends on Facebook. In the current experiment, the Likes reflected aggregated feedback from unknown users, which is possibly less meaningful than observing Likes from friends, acquaintances, or well-known persons. However, an alternative explanation is that the Likes were not perceived and processed as social cues in the first place. In other words, the numbers indicating the number of Likes were not processed as cues for positive affectivity; instead, they were processed as mere numbers without a specific meaning.

The study by Winter and colleagues (2015) also did not yield a significant effect of Likes when comparing the high and low number of Likes (instead of presence and omission of Likes in the current study). In their discussion, they speculate that due to a negativity bias Likes do not arouse attention, because they are limited to positivity. Secondly, they speculate that Likes have less influence on the readers, as they are less concrete than comments. Kluck and colleagues (2019) made a similar observation. They investigated the influence of a bandwagon credibility rating (i.e., a cue of the average credibility score from a large group of users), which also failed to influence the readers. Their explanation was, just as Winter et al., that the aggregated user feedback is more ambiguous than comments. Both studies refer to the exemplification theory by Zillmann (1999) and stipulate that comments, which are more concrete, are better exemplars of peer-user attitudes than the aggregated user feedback. Therefore, comments are supposedly processed with less cognitive effort than Likes.

Thus, as Winter et al. and Kluck et al. have previously proposed, Likes are probably interpreted and processed differently than the comments. Likes indicate popularity in a quantitative measure. A quantity or number is possibly not processed as intuitively as the rhetoric in the comment section. In other words, numbers are possibly less meaningful to the reader, because they do not convey explicit semantics or ideologies. In line with this notion, Likes have been conceptualized as paralinguistic digital affordances, which allegedly do not convey a specific predefined meaning (Hayes et al., 2016). Moreover, studies have shown that numbers are sometimes not intuitively processed as meaningful affective cues. For example, when a data display presents a large number of human casualties to an audience, this number does not engender equally representative feelings of empathy in this audience (Slovic et al., 2011). Campbell and Offenhuber (2019) have stated "numbers representing lives do not convey the importance of those lives-numbers numb". Perhaps, social media users do not implicitly attribute meaning to the number of Likes just as human casualty numbers fail to induce an appropriate emotional response in readers. Comments, on the other hand, are more concrete exemplars of peer-user attitudes and therefore are more influential (Kluck et al., 2019; Winter et al., 2015).

In addition to exemplification theory, dual processing system theories may provide a useful framework for the processing of Likes and comments (Barrett et al., 2004; Epstein, 1994). If social media users employ an analytic, slow, and reflective information processing system (i.e., system 2), then the underlying meaning of the number of Likes, which is the positive affectivity of other users, can be inferred. However, if social media users employ a fast, intuitive, emotionally driven system, that uses fewer cognitive resources (i.e., system 1), they are less likely to ascribe explicit meaning to the number of Likes. In that case, social media users are less likely to utilize the Likes to form their personal opinions. Comments, on the other hand, are more concrete exemplars of peer opinions, which are comprehended without the need for analytic and inferential information processes. Future research should investigate which underlying mechanisms are involved in the perception, interpretation, and utilization of Likes.

A limitation of our experiment was that the assumed ideological disposition of the readers or their attitude towards the article content was different than initially expected. That is, participants were less negative about the ideologically incongruent article content (i.e., an article about the relationship between video 
games and aggressive behaviour) than initially assumed. Ideally, participants would show a more negative attitude toward the incongruent article content than the neutral article-content, in order to examine the interaction of personal disposition and comment sentiment. That said, we do not think this limitation has drastically confounded our general findings; positive comments appeared to have little effect on the readers' attitude, which makes an interaction effect between a negative disposition and positive comments unlikely.

In conclusion, beliefs and opinions about news presented on social media can easily be affected by negative peer-user comments. Most strikingly, the evaluation of ostensibly objective information can be distorted by subjective, emotive, and fallacious rhetoric. Likes, on the other hand, do not appear to influence the readers as much. The current findings contribute to the psychology of online media behaviour and may have some important implications. If a news platform aims to objectively inform its audience, the findings of this study may serve as an important consideration on whether comment sections should be included on the platform's news pages. In addition, social media users may benefit by being more aware of the potential influence of peer-user comments on their personal beliefs and attitudes. One should ask: 'does the internet stranger who wrote this comment actually have authoritative experience or knowledge on this topic?' While critical and negative comments may appear convincing, it is wise to be sceptical about the criticisms.

\section{Data availability}

The data and codes used for analyses can be found on OSF (available at https://doi.org/10.17605/OSF.IO/MQ2G8).

Received: 8 October 2020; Accepted: 1 September 2021;

Published online: 10 September 2021

\section{Note}

1 An experimental study of online social cues; how Likes and peer-user comments influence the processing of social-media content: https://osf.io/d37f5.

\section{References}

Appelman A, Sundar SS (2016) Measuring Message Credibility. Journal Mass Commun Q 93(1):59-79. https://doi.org/10.1177/1077699015606057

Asch SE (1951) Effects of group pressure on the modification and distortion of judgments. In: Guetzkow HS (ed) Groups, leadership and men; research in human relations. Carnegie Press, Pittsburgh, pp. 177-190

Asch SE (1955) Opinions and social pressure. Sci Am 193(5):31-35. https://doi.org/ 10.1038/scientificamerican1155-31

Asch SE (1956) Studies of independence and conformity: I. A minority of one against a unanimous majority. Psychol Monogr-Gen A 70(9):1-70. https:// doi.org/10.1037/h0093718

Bak MP, Keßler T (2012) I like it if you like it! Conformity effects on Facebook J Bus Media Psychol 3(2):23-30

Barrett LF, Tugade MM, Engle RW (2004) Individual differences in working memory capacity and dual-process theories of the mind. Psychol Bull 130(4):553-573. https://doi.org/10.1037/0033-2909.130.4.553

Baumeister R, Bratslavsky E, Finkenauer C et al. (2001) Bad is stronger than good. Rev Gen Psychol 5(4):323-370

Cacioppo JT, Petty RE (1982) The need for cognition. J Pers Soc Psychol 42:116-131

Campbell S, Offenhuber D (2019) Feeling numbers: the emotional impact of proximity techniques in visualization. Inf Des J 25(1):71-86. https://doi.org/ 10.1075/idj.25.1.06cam

Carr CT, Wohn DY, Hayes RA (2016) As social support: relational closeness, automaticity, and interpreting social support from paralinguistic digital affordances in social media. Comput Hum Behav 62:385-393

Carstensen LL, DeLiema M (2018) The positivity effect: a negativity bias in youth fades with age. Curr Opin Behav Sci 19:7-12. https://doi.org/10.1016/ j.cobeha.2017.07.009

Epstein S (1994) Integration of the cognitive and the psychodynamic unconscious. Am Psychol 49(8):709-724. https://doi.org/10.1037/0003-066X.49.8.709/ 0003-066X.49.8.709
Eranti V, Lonkila M (2015) The social significance of the Facebook Like button. First Monday 20(6) https://doi.org/10.5210/fm.v20i6.5505

Facebook to hide number of likes in trial aimed at improving users' wellbeing (2019). Australian Associated Press. Retrieved from https://www.theguardian.com/ technology/2019/sep/27/facebook-to-hide-number-of-likes-in-trial-aimed-atimproving-users-wellbeing.

Gaming penetration in the United Kingdom (UK) from 2013 to 2021, by age group and gender (2021). Statista. https://www.statista.com/statistics/300513/gamingby-demographic-group-uk/ Accessed 23 Jul 2021

Habernal I, Gurevych I (2016) Which argument is more convincing? Analyzing and predicting convincingness of Web arguments using bidirectional LSTM. In: Erk K, Smith NA (eds) Proceedings of the 54th annual meeting of the Association for Computational Linguistics (ACL), Berlin, 2016

Hayes RA, Carr CT, Wohn DY (2016) One click, many meanings: Interpreting paralinguistic digital affordances in social media. J Broadcast Electron 60(1):171-187

Hinnant A, Subramanian R, Young R (2016) User comments on climate stories: impacts of anecdotal vs. scientific evidence. Clim Change 138(3-4):41-424

Inbar Y, Lammers J (2012) Political diversity in social and personality psychology. Perspect Psychol Sci 7(5):496-503. 10.1177\%2F1745691612448792

Ito TA, Larsen JT, Smith NK et al. (1998) Negative information weighs more heavily on the brain: the negativity bias in evaluative categorizations. J Pers Soc Psychol 75(4):887

Johnston WA, Hawley KJ, Plewe SH et al. (1990) Attention capture by novel stimuli. J Exp Psychol Gen 119(4):397-411. https://doi.org/10.1037/00963445.119.4.397

Kim JW (2018) They liked and shared: effects of social media virality metrics on perceptions of message influence and behavioural intentions. Comput Hum Behav 84:153-161

Kluck JP, Schaewitz L, Krämer NC (2019) Doubters are more convincing than advocates. The impact of user comments and ratings on credibility perceptions of false news stories on social media. Stud Commun Media 8(4):446-470

Lakens D (2014) Performing high-powered studies efficiently with sequential analyses. Eur J Soc Psychol 44(7):701-710. https://doi.org/10.1002/ejsp.2023

Lazer DMJ, Baum MA, Benkler Y et al. (2018) The science of fake news. Science 359:1094-1096. https://doi.org/10.1126/science.aao2998

Lowe-Calverley E, Grieve R (2018) Thumbs up: a thematic analysis of image-based posting and liking behaviour on social media. Telemat Inform 35(7):1900-1913

Milgram S (1963) Behavioral study of obedience. J Abnorm Soc Psych 67(4):371

Mutz DC (1998) Impersonal influence: how perceptions of mass collectives affect political attitudes. Cambridge University Press, Cambridge

Nadeau R, Cloutier E, Guay JH (1993) New evidence about the existence of a bandwagon effect in the opinion formation process. Int Polit Sci Rev $14(2): 203-213$

Norris CJ (2021) The negativity bias, revisited: evidence from neuroscience measures and an individual differences approach. Soc Neurosci 16(1):68-82. https://doi.org/10.1080/08824096.2021.1915269

Ozanne M, Cueva Navas A, Mattila AS et al. (2017) An investigation into Facebook "Liking" behavior an exploratory study. Soc Media Soc 3(2):1-12. 10.1177\% 2F2056305117706785

Pohl D, Choi A, Marcus K et al. (2021) Political party preference of freshmen university students and its association with student lifestyle characteristics and the influence of 1 year public university. J Child Adolesc Health 5(2):1-8

Rodriguez S (2019) Instagram may stop showing how many people 'like' each post. CNBC. https://www.cnbc.com/2019/04/30/instagram-hiding-like-counts-intest.html. Accessed 16 Apr 2020

Rozin P, Royzman EB (2001) Negativity bias, negativity dominance, and contagion. Pers Soc Psychol Rev 5(4):296-320

Schmitt-Beck R (2015) Bandwagon effect. In: Mazzoleni G (ed) The international encyclopedia of political communication. Wiley-Blackwell, Hoboken, pp. 1-5

Sherman LE, Payton AA, Hernandez LM et al. (2016) The power of the like in adolescence: effects of peer influence on neural and behavioral responses to social media. Psychol Sci 27(7):1027-1035

Slovic P, Zionts D, Woods AK et al. (2011) Psychic numbing and mass atrocity. In: Shafir E (ed) The behavioral foundations of public policy. Princeton University Press, Princeton, pp. 126-142

Sundar SS (2008) The MAIN model: a heuristic approach to understanding technology effects on credibility. In: Metzger MJ, Flanagin AJ (eds) Digital media, youth, and credibility. The MIT Press, Cambridge, pp. 72-100

Thorson K, Vraga E, Ekdale B (2010) Credibility in Context: How Uncivil Online Commentary Affects News Credibility. Mass Commun Soc 13(3):289-313. https://doi.org/10.1080/15205430903225571

Waddell TF (2018a) This tweet brought to you by a journalist: How comment gatekeeping influences online news credibility. Electron News 12(4):218-234

Waddell TF (2018b) What does the crowd think? How online comments and popularity metrics affect news credibility and issue importance. New Media Soc 20(8):3068-3083

Winter S, Brückner C, Krämer NC (2015) They came, they liked, they commented: social influence on Facebook news channels. Cyberpsych Beh Soc 18(8):431-436 
Xu Q (2013) Social recommendation, source credibility, and recency: effects of news cues in a social bookmarking website. J Mass Commun Q 90(4):757-775

Zillmann D (1999) Exemplification theory: judging the whole by some of its parts. Media Psychol 1(1):69-94. https://doi.org/10.1207/s1532785xmep0101_5

\section{Competing interests}

The authors declare no competing interests.

\section{Additional information}

Supplementary information The online version contains supplementary material available at https://doi.org/10.1057/s41599-021-00889-5.

Correspondence and requests for materials should be addressed to Arnout B. Boot.

Reprints and permission information is available at http://www.nature.com/reprints
Publisher's note Springer Nature remains neutral with regard to jurisdictional claims in published maps and institutional affiliations.

\begin{abstract}
(c) Open Access This article is licensed under a Creative Commons Attribution 4.0 International License, which permits use, sharing, adaptation, distribution and reproduction in any medium or format, as long as you give appropriate credit to the original author(s) and the source, provide a link to the Creative Commons license, and indicate if changes were made. The images or other third party material in this article are included in the article's Creative Commons license, unless indicated otherwise in a credit line to the material. If material is not included in the article's Creative Commons license and your intended use is not permitted by statutory regulation or exceeds the permitted use, you will need to obtain permission directly from the copyright holder. To view a copy of this license, visit http://creativecommons.org/ licenses/by/4.0/.
\end{abstract}

(C) The Author(s) 2021, corrected publication 2021 